\section{Pólipos gastrointestinales en pediatría}

\section{RESUMEN}

Antecedentes: el pólipo gastrointestinal es un tumor o crecimiento localizado que protruye desde la pared hacia la luz intestinal. Su manifestación clínica más común es la rectorragia recurrente; también puede causar dolor abdominal, prolapso del pólipo por el recto, prurito, dolor después de la defecación, diarrea mucosa o constipación de vientre. La colonoscopia es el procedimiento de elección para diagnosticar y tratar los pólipos.

Objetivo: describir las características clínicas, colonoscópicas e histológicas de los pacientes pediátricos con poliposis colónica, atendidos en el Servicio de Gastroenterología y Nutrición del Instituto Nacional de Pediatría, México.

Materiales y métodos: estudio observacional, transversal, retrospectivo, descriptivo. Se revisaron todos los expedientes de los pacientes a quienes se realizó estudio endoscópico del 4 de junio del 2010 al 31 de diciembre del 2013.

Resultados: se revisaron 56 expedientes; 49 cumplían los criterios de inclusión con predominio del sexo masculino (59.2\%). El síntoma principal de estos pacientes fue el sangrado moderado del tubo digestivo bajo no anemizante en 41 casos $(83.7 \%)$; la localización más frecuente fue rectal $(51 \%)$.

Conclusiones: en pacientes preescolares y escolares con hemorragia de tubo digestivo bajo "no anemizante", la primera posibilidad diagnóstica es un pólipo que debe ser diagnosticado, tratado y, en su caso, seguido por el tiempo necesario.

Palabras clave: poliposis intestinal, sangrado de tubo digestivo bajo, colonoscopia.

\section{Gastrointestinal polyps in pediatrics}

\author{
ABSTRACT \\ Background: Gastrointestinal polyp refers to a localized tumor or growth \\ that protrudes through the lumen of the gastrointestinal tract. The most \\ common clinical presentation is recurrent rectal bleeding; as well it \\ could be associated with abdominal pain, prolapsed polyp, pruritus, \\ pain after defecation or constipation. Colonoscopy is the procedure of \\ choice for diagnosis and treatment.
}

Josefina Monserrat Cázares-Méndez Vianey Paola Zamudio-Vázquez

Esteban Gómez-Morales

Silvia Guadalupe Ortiz-Aguirre

José Francisco Cadena-León

Erick Manuel Toro-Monjaraz

Erika Montijo-Barrios

Flora Zárate-Mondragón

Roberto Cervantes-Bustamante

Jaime Alfonso Ramírez-Mayans

Martha Verónica López-Ugalde

Servicio de Gastroenterología y Nutrición, Instituto Nacional de Pediatría, México.

Recibido: 15 de enero del 2015

Aceptado: 20 de abril del 2015

Correspondencia: Dra. Vianey Paola Zamudio Vázquez

Depto. de Gastroenterología y Nutrición Pediátrica Instituto Nacional de Pediatría

Insurgentes Sur 3700- C

CP 04530 México, D.F.

Teléfono: 10840900 ext. 1288

paolazamudiovazquez@gmail.com

Este artículo debe citarse como

Cázares-Méndez JM, Zamudio-Vázquez VP, GómezMorales E, Ortiz-Aguirre SG, Cadena-León JF, ToroMonjaraz EM et al. Pólipos gastrointestinales en pediatría. Acta Pediatr Mex 2015;36:158-163. 
Objective: Describe clinical manifestations colonoscopy and histological characteristics in pediatric population with colonic polyps, studied at the Department of Gastroenterology and Nutrition of our institution.

Methods: A retrospective observational, descriptive, cross-sectional study was conducted. We reviewed the case records of all the patients that were submitted to endoscopic procedures between June 4, 2010 to December 31, 2013.

Results: We reviewed 56 clinical records, 49 met the inclusion criteria. Male patients (59.2\%); the main symptom was lower intestinal hemorrhage (83.7\%); the most common site was rectal in $51 \%$.

Conclusions: In pre-school and school-age patients with non anemic lower intestine hemorrhage, the main diagnose is an intestinal polyp, which should be treated and followed as long as necessary.

Key words: Intestinal polyp, Lower intestinal hemorrhage, Colonoscopy.

\section{INTRODUCCIÓN}

Un pólipo gastrointestinal es un tumor o crecimiento localizado que protruye desde la pared hacia la luz intestinal. Los pólipos se pueden clasificar desde el punto de vista morfológico en pediculados o sésiles, también en neoplásicos o no neoplásicos (Cuadro 1). ${ }^{1}$

Su número puede ser muy variable; cuando son pocos (menos de 3) suelen ser esporádicos pero si son numerosos (más de 3) se habla de poliposis intestinal. ${ }^{1,2}$ Cuando existen múltiples pólipos en el tracto gastrointestinal y se acompañan de una enfermedad genética, su histopatología, heredabilidad y el aumento del riesgo de cáncer gastrointestinal o en otros órganos es

Cuadro 1. Características neoplásicas de los pólipos

\begin{tabular}{ll}
\hline Neoplásicos & No neoplásicos \\
Adenomas benignos & Hamartomatosos o pólipos juveniles \\
Carcinomas malignos & $\begin{array}{r}\text { Hiperplásicos } \\
\text { Inflamatorios }\end{array}$
\end{tabular}

diferente de acuerdo con la afección de que se trate. Se reconocen dos grupos mayores de poliposis: síndromes de poliposis adenomatosa (poliposis adenomatosa familiar, síndrome de Gardner y síndrome de Turcot y otros) y poliposis hamartomatosa (poliposis juvenil, síndrome de Bannayan-Riley-Ruvalcaba, enfermedad de Cowden, síndrome de Peutz-Jeghers); otros son: síndromes de poliposis mixta y poliposis hiperplásica. ${ }^{1}$ Se presentan en 3 a $4 \%$ de la población menor de 21 años y en $1 \%$ de la población de preescolares y escolares; son la causa más frecuente de hemorragia del tubo digestivo bajo no anemizante en este grupo de edad. En 70 a $80 \%$ de los casos son únicos y se encuentran distales, en recto sigmoides. Los pólipos juveniles, sean únicos o múltiples (> 3) son las lesiones polipoides más frecuentes en la edad pediátrica, hasta en $97 \%$ de los casos; $90 \%$ son pediculados y miden de 1 a $2 \mathrm{~cm}$ de diámetro. La edad más frecuente de presentación es la primera década de la vida, con una incidencia mayor entre los 2 y los 6 años de edad. Son muy raros en el primer año de vida y después de los 10 años de edad. ${ }^{1-6}$ Son más frecuentes en el sexo 
masculino y en muchos casos son asintomáticos; sin embargo, la presentación clínica más común es la rectorragía recurrente, que acompaña a la defecación; también pueden asociarse con dolor abdominal, prolapso del pólipo por recto, prurito, dolor después de la defecación, diarrea mucosa o constipación de vientre. ${ }^{1,3}$

Rara vez causan anemia aguda, a menos que el pólipo se autoescinda y hasta en un tercio de los casos puede haber anemia por deficiencia de hierro. ${ }^{1}$ Las manifestaciones fenotípicas extracolónicas se encuentran bien documentadas en el caso del síndrome de Peutz-Jeghers, así como en la poliposis adenomatosa familiar; incluso pueden orientar al diagnóstico antes de contar con el informe histopatológico. Sin embargo, en la poliposis juvenil estas anormalidades no han podido definirse claramente..$^{6-8}$

La colonoscopia es el procedimiento de elección para diagnosticar los pólipos ya que, además del diagnóstico, permite su exéresis. ${ }^{1-3}$ Actualmente se recomienda que se realice una revisión total del colon, ya que si bien es más frecuente encontrarlos en recto sigmoides también pueden hallarse en zonas más proximales o en mayor cantidad. ${ }^{3}$

El tratamiento es la exéresis durante la colonoscopia. Los pólipos pediculados menores de $5 \mathrm{~mm}$ pueden resecarse con asa fría, los mayores, con asa caliente, con electrocauterio monopolar. Los pólipos pediculados con tallo mayor de $10 \mathrm{~mm}$ deben ser resecados con asas desprendibles (Endoloops, Olympus, Japón) por tener mayor riesgo de perforación y hemorragia. Después de su resección, los pólipos juveniles recurren en 4 a $7 \%$ de los pacientes. ${ }^{1,3}$

El tejido obtenido debe enviarse a revisión histopatológica ya que, en caso de sospecha de malignidad o enfermedad sindromática, se debe dar seguimiento endoscópico de manera periódi- ca, así como estudio genético de los familiares y de los pacientes para analizar las características que orienten hacia un síndrome determinado.

\section{OBJETIVO}

El objetivo de este estudio es describir las características clínicas, colonoscópicas e histológicas de los pólipos colónicos en pacientes pediátricos atendidos en el Servicio de Gastroenterología y Nutrición del Instituto Nacional de Pediatría (México), así como las principales indicaciones para una colonoscopia y para determinar complicaciones.

\section{MATERIAL Y MÉTODOS}

Estudio observacional, transversal, retrospectivo y descriptivo. Se revisaron todos los expedientes de los pacientes a quienes se les realizó estudio endoscópico del 4 de junio del 2010 al 31 de diciembre del 2013 y en quienes se encontraron uno o más pólipos. Se recolectaron las siguientes variables: edad, sexo, síntomas, localización del pólipo, tipo de pólipo, tipo de resección del mismo, histología y complicaciones. Se excluyeron los pacientes cuyos expedientes estaban incompletos.

Los resultados obtenidos se analizaron mediante el programa SPSS versión 17.0. Para el análisis estadístico se utilizó estadística descriptiva, para variables numéricas medidas de tendencia central, media y desviación estándar o mediana y valores mínimo y máximo, de acuerdo con la presencia o no de normalidad; para las variables categóricas frecuencias y proporciones.

\section{RESULTADOS}

De un total de 56 expedientes 49 cumplían con los criterios de inclusión. Predominó el sexo masculino: 29 pacientes (59.2\%). La edad media al diagnóstico fue de 7 años con una desviación 
estándar de \pm 4 años; la edad menor fue de 1 año en 4 pacientes $(8.2 \%)$ y la mayor de 16 años en 2 pacientes $(4.1 \%$ ) con un rango de 12 años; 10 pacientes (20.4\%) fueron mayores de 10 años (Cuadro 2).

El síntoma principal fue sangrado de tubo digestivo bajo no anemizante (rectorragia) en 41 casos $(83.7 \%)$, de los cuales sólo en $2(4 \%)$ se palpó masa en el recto; en 4 (8\%) hubo dolor abdominal y en 3 de ellos el dolor acompañaba a la rectorragia, en uno fue el único síntoma; en 1 caso $(2 \%)$ hubo prolapso rectal; en 5 pacientes $(10.2 \%)$ se realizó endoscopia por antecedentes familiares de poliposis intestinal o manifestaciones extraintestinales (manchas en boca) (Cuadro 3).

En 19 casos sólo se realizó colonoscopia, en 25 panendoscopia y colonoscopia y en 5 se efectuó únicamente endoscopia alta debido a hipertensión portal (2 casos, sin sangrado de tubo digestivo, solo seguimiento de su enfermedad de base), sangrado de tubo digestivo distal (2 casos) y dolor abdominal crónico en un caso. En 27 casos (55.1\%) se encontró poliposis única, en 4

Cuadro 2. Distribución de acuerdo con la edad

\begin{tabular}{ccc}
\hline Edad (años) & Número de pólipos & $\mathbf{( \% )}$ \\
1 & 4 & 8.2 \\
2 & 1 & 2 \\
3 & 4 & 8.2 \\
4 & 5 & 10.2 \\
5 & 4 & 8.2 \\
6 & 5 & 10.2 \\
7 & 3 & 6.1 \\
8 & 7 & $\mathbf{1 4 . 3}$ \\
9 & 2 & 4.1 \\
10 & 4 & 8.2 \\
11 & 3 & 6.1 \\
12 & 2 & 4.1 \\
14 & 2 & 4.1 \\
15 & 1 & 2 \\
16 & 2 & 4.1 \\
Total & 49 & 100 \\
& & \\
\end{tabular}

Cuadro 3. Principales síntomas e indicación de estudio colonoscópico

\begin{tabular}{|c|c|c|}
\hline Síntomas & $\begin{array}{l}\text { Número } \\
\text { de casos }\end{array}$ & $(\%)$ \\
\hline $\begin{array}{l}\text { Sangrado de tubo digestivo bajo no } \\
\text { anemizante }\end{array}$ & 36 & 73.5 \\
\hline $\begin{array}{l}\text { Sangrado de tubo digestivo bajo no ane- } \\
\text { mizante y dolor abdominal }\end{array}$ & 3 & 6.1 \\
\hline $\begin{array}{l}\text { Antecedentes familiares y manchas en } \\
\text { la boca }\end{array}$ & 3 & 6.1 \\
\hline Antecedentes familiares & 2 & 4.1 \\
\hline $\begin{array}{l}\text { Sangrado de tubo digestivo bajo no } \\
\text { anemizante y masa palpable en recto }\end{array}$ & 2 & 4.1 \\
\hline Prolapso rectal & 1 & 2 \\
\hline Dolor abdominal & 1 & 2 \\
\hline $\begin{array}{l}\text { Constipación de vientre y antecedentes } \\
\text { familiares }\end{array}$ & 1 & 2 \\
\hline Total & 49 & 100 \\
\hline
\end{tabular}

se encontraron dos pólipos y en 18, tres o más (36.7\%) (Cuadro 4).

La localización más frecuente de los pólipos únicos fue rectal en 25 casos (51\%). Cuando la poliposis era múltiple las localizaciones más frecuentes fueron: recto en 32 casos $(65.3 \%)$, gástrica en 13 casos (26.5\%), colon transverso en 8 casos $(16.3 \%)$, sigmoides y en la primera porción del duodeno 7 casos (14.2\%) cada uno. La mediana del diámetro del pólipo fue de $0.5 \mathrm{~cm}$, con mínimo de $0.5 \mathrm{~cm}$ y un máximo de $3 \mathrm{~cm}$ y un rango de $1.5 \mathrm{~cm}$; en 34 casos $(69.4 \%)$ los pólipos fueron pediculados, en 11 (22.4\%) sésiles y en $4(8.2 \%)$, ambos.

Cuadro 4. Distribución de número de pólipos por paciente

\begin{tabular}{ccc}
\hline Número de pólipos & Número de casos & $\mathbf{( \% )}$ \\
1 & 27 & 55.1 \\
2 & 4 & 8.2 \\
3 & 6 & 12.2 \\
4 & 3 & 6.1 \\
6 & 2 & 4.1 \\
7 & 1 & 2 \\
8 & 1 & 2 \\
10 & 3 & 6.1 \\
20 o más & 2 & 4.1 \\
Total & 49 & 100 \\
\hline
\end{tabular}


De 49 pacientes 11 casos (22.4\%) tenían síndrome de poliposis juvenil, 10 (20.4\%) síndrome de Peutz-Jeghers y 4 (8.2\%) síndrome de BannayanRiley-Ruvalcaba.

\section{Técnica de extracción}

Se utilizó asa caliente en la mayoría de los procedimientos $(38,77.6 \%)$ y todos fueron pólipos pediculados; en 11 (22.4\%) se realizó toma de biopsia con pinza fría debido a que se trataba de pólipos sésiles; 2 casos (4.1\%) en los que se realizó polipectomía con asa caliente hubo complicaciones: un paciente síndrome con pospolipectomía y otro con perforación colónica.

El dato histológico fue: pólipos hamartomatosos en 42 casos (85.7\%) y en algunos casos se reportaron histologías mixtas (Cuadro 5). Se debe tener vigilancia para algunos casos, especialmente los sindromáticos.

\section{DISCUSIÓN}

En este estudio la mayoría de los datos demográficos estudiados concuerdan con los reportados en la literatura especializada; tal es el caso de la edad media descrita ( 6.8 años) que como en nuestro estudio fue de 7.07 años. En cuanto al rango de edad se observó una variación, pues a diferencia de lo descrito en las revisiones, que varía de 2 hasta 8 años, nosotros encontramos un rango de edad de presentación de uno hasta 15 años. Llama la atención que en los reportes publicados se comenta que los casos de pólipos

Cuadro 5. Tipos histológicos de pólipos

\begin{tabular}{lcc}
\hline Tipo histológico & Número de casos & $\mathbf{( \% )}$ \\
Hamartomatoso & 42 & 85.7 \\
Inflamatorio & 1 & 2 \\
Hiperplásico & 3 & 6.2 \\
Hamartomatoso e inflamatorio & 2 & 4.1 \\
Inflamatorio e hiperplásico & 1 & 2 \\
Total & 49 & 100
\end{tabular}

en niños menores de un año y mayores de 10 años se da de manera esporádica. En nuestro estudio los pacientes con edad de un año representaron $8.4 \%$ de los casos y los mayores de 10 años $20.4 \%$; $^{1-6}$ situación que podría deberse a que nuestro hospital es un centro nacional de referencia.

En nuestro estudio, coincidió también el ligero predominio de aparición en el sexo masculino, con una relación de 1.2:1, aunque algunos autores han reportado una proporción de 3:2. ${ }^{9}$

El pólipo juvenil continua siendo en esta edad el tipo más frecuente, tal como se describe en múltiples revisiones, así como también en un artículo previo realizado en este hospital donde se encontró una frecuencia de $94.9 \% .^{10}$ La localización de los pólipos en ese estudio, como en éste y en el resto de los publicados, fue en los primeros $10 \mathrm{~cm}$ del margen anal.

Después de que se introdujo la endoscopia flexible se han diagnosticado más casos de pólipos gástricos en los que los pacientes presentan sangrado del tubo digestivo alto y dolor abdominal. Su importancia reside en el riesgo de malignización de este tipo de pólipos, por lo que se recomienda su resección. ${ }^{11}$ Sin embargo, el tratamiento de los pólipos gástricos aislados y pequeños aún sigue siendo motivo de controversia.

En nuestro estudio $10.2 \%$ de los pólipos se haIlaban exclusivamente en estómago, similar a lo encontrado en el estudio realizado por Li-Chun Wang ${ }^{11}$ en el que, de 50 pacientes, 4 presentaron pólipo gástrico aislado y sólo se reportó la histología de 3 de ellos: inflamación crónica, hiperplasia y uno relacionado con Helicobacter pylori; a diferencia de nuestro estudio en el que los pólipos gástricos encontrados fueron asociados principalmente con una enfermedad sindromática como Peutz- Jeghers y BannayanRiley-Ruvalcaba. Un hallazgo en un caso fue 
dolor abdominal crónico recurrente en un paciente con hipertensión portal; en un niño con fibrosis quística que presentaba sangrado del tubo digestivo alto y en una niña de 17 años con sangrado del mismo tipo.

En esta revisión se encontraron 2 pacientes (4.1\%) con complicaciones debidas al procedimiento endoscópico: uno de ellos con síndrome pospolipectomía y uno más (2\%) con perforación. Esta última coincide con el porcentaje de complicaciones informado por Macrae ${ }^{12}$ en el que el porcentaje de perforaciones en un estudio de 5000 colonoscopias para diagnóstico o terapéuticas fue de 0 a $3 \%$.

La indicación de colonoscopia en estos pacientes fue en $73.5 \%$ sangrado del tubo digestivo bajo no anemizante. En el estudio realizado previamente en este hospital (Cervantes 2001) ${ }^{5}$ se sugería que en presencia de sangrado del tubo digestivo bajo no anemizante la colonoscopia debía reservarse para pacientes sin evidencia de pólipo mediante tacto rectal o rectosigmoidoscopia. Sin embargo, actualmente se considera la colonoscopia como método de diagnóstico de primera elección debido a que se puede explorar el colon en su totalidad, para detectar pólipos que se encuentren más allá de la localización más frecuente que son los 10 primeros centímetros del margen anal, permitiendo su exéresis.

\section{CONCLUSIONES}

Los pólipos gastrointestinales en niños son mayoritariamente benignos, de presencia esporádica y sin secuelas a largo plazo. Sin embargo, también los hay asociados con enfermedades sindromáticas que requerirán tratamiento y seguimiento; así como otros que deben ser detectados y tratados tempranamente pues tienen alto riesgo de malignización. Por lo tanto, en todo paciente preescolar y escolar con hemorragia del tubo digestivo bajo "no anemizante" el pólipo debe considerarse como la primera posibilidad diagnóstica y tiene que ser diagnosticado, tratado y, en su caso, seguido a través del tiempo necesario.

\section{REFERENCIAS}

1. Mougenot JF, Olshwang S, Peuchmar M. Intestinal Tumors: Intestinal Polyps and Polyposis. En Walker: Pediatric Gastrointestinal Disease: Pathology, Diagnosis, Management. Volume 1. 4th Edition. 2004. p.214-223

2. Andreu M. Pólipos colorrectales y poliposis intestinal. [Internet] España: Elsevier. [07 Abril 2015]. Disponible en: http://www.elsevierinstituciones.com/ficheros/booktemplate/9788475927220/files/Capitulo30.pdf

3. Gee Lee B. Juvenile Polyp and Colonoscopic Polypectomy in Childhood. J Pediatr Gastroenterol Nutr 2012;15(4):250255.

4. Livinstone EM, Troncale FJ, Sheahan DG. Value of a single fórceps biopsy of colonic polyps. Gastroenterology 1977;73:1296-8.

5. Cervantes-Bustamante R, Ramirez-Mayans J, Mata-Rivera $\mathrm{N}$, Cuevas-Schat. Juvenile polyposis in Mexican children. Rev Gastroenterol Mex 2002;67:150-4.

6. Latt TT, Nichell R, Domizio P. Rectal bleeding and polyps. Arch Dis Child 1993;69:144-7.

7. Desai D, Murday V, Phillips R, Neale K, Milla P, Hudgson S. A survey phenotypic features in juvenile polyps. J Med Genet 1998;35:476-81.

8. Dean P. Hereditary intestinal polyposis syndromes. Rev Gastroenterol Mex 1996;61:100-11.

9. Winter HS. Intestinal polyps. In: Spiro H.M editor. Clinical gastroenterology. 5th ed. New York: McGraw-Hill; 1993, p. 777-9.

10. Ramírez MJ, Rivera EM. Coronado ME. Pólipos de recto y colon en niños. Bol Med Hosp Inf Mex 1984;41:437-41.

11. Li-Chun Wang. Gastrointestinal Polyps in Children. Pediatr Neonatol 2009;50(5):196-201.

12. Macrae FA, Tan KG, Willians CB. Towards safer colonoscopy: a report on the complications of 5000 diagnostic or therapeutic colonoscopies. Gut 1983;24:376-83. 